\title{
The robustness of the galaxy distribution function to effects of merging and evolution
}

\author{
Abel Yang \\ Department of Astronomy, University of Virginia, Charlottesville, VA 22904 \\ William C. Saslaw \\ Institute of Astronomy, Madingley Road, Cambridge CB3 OHA, UK; and Department of \\ Astronomy, University of Virginia, Charlottesville, VA 22904 \\ Aik Hui Chan \\ Department of Physics, National University of Singapore, 2 Science Drive 3, Singapore 117542; \\ and Institute of Advances Studies, Nanyang Technological University, \#02-18 60 Nanyang View, \\ Singapore 639673 \\ Bernard Leong \\ Senatus Pte. Ltd, 19 Amoy Street, 02-01, Singapore 069584
}

\begin{abstract}
We examine the evolution of the spatial counts-in-cells distribution of galaxies and show that the form of the galaxy distribution function does not change significantly as galaxies merge and evolve. In particular, bound merging pairs follow a similar distribution to that of individual galaxies. From the adiabatic expansion of the universe we show how clustering, expansion and galaxy mergers affect the clustering parameter $b$. We also predict the evolution of $b$ with respect to redshift.
\end{abstract}

Subject headings: galaxies: statistics — cosmology: theory — large-scale structure of universe

\section{Introduction}

The galaxy spatial distribution function $f(N, V)$ is a simple but powerful statistic which characterizes the locations of galaxies in space. It includes statistical information on voids and other underdense regions, on clusters of all shapes and sizes, on filaments, on the probability of finding an arbitrary number of neighbors around randomly located positions, on counts of galaxies in cells of arbitrary shapes and sizes randomly located, and on galaxy correlation functions of all orders. These are just some of its representations (Saslaw 2000; Saslaw \& Yang 2010). Moreover it is also 
closely related to the distribution function of the peculiar velocities of galaxies around the Hubble flow (Saslaw et al. 1990; Leong \& Saslaw 2004).

A physically motivated form of the distribution function for galaxies in quasi-equilibrium was derived and generalized using a statistical mechanical approach. (Ahmad et al. 2002, 2006b) It has also been generalized to systems containing particles of two different masses (Ahmad et al. 2006a). In its simplest form, the probability of finding $N$ galaxies in a cell of volume $V$ is given by the gravitational quasi-equilibrium distribution(GQED):

$$
f_{V}(N)=\frac{\bar{N}(1-b)}{N !}(\bar{N}(1-b)+N b)^{N-1} \exp (-\bar{N}(1-b)-N b)
$$

where $\bar{N}$ is the average number of galaxies in a cell and the clustering parameter $b=-W / 2 K$ is the ratio of the gravitational correlation energy $W$ to twice the kinetic energy $K$ of peculiar velocities relative to the Hubble flow. Although other distributions have been proposed (e.g. negative binomial; for an early review see Fry 1986), they are generally not physically motivated. Extensive computer simulations (summarized in Saslaw 2000) designed to test the GQED agree closely with the analytical results. Observations at both low (Sivakoff \& Saslaw 2005) and high (Rahmani et al. 2009) redshifts suggest that the form of the distribution function $f_{V}(N)$ is essentially unchanged over a wide range of redshifts. However, mergers among galaxies could have modified the form of $f_{V}(N)$ significantly compared to the simple model which involves no mergers at all, and yet they did not. In this paper we examine the robustness of the GQED to merging galaxies.

\section{The positions of merging galaxy pairs}

Merging galaxy pairs are often extended structures that would have a different interaction potential than simple spheres or point masses. With a different interaction potential, they may also be distributed differently. Extended structures resulting from mergers will not only have a different interaction potential, they also have a different mass and should be treated differently. To consider how these extended structures influence the GQED, we first consider how they modify the interaction potential. Using the modified potential we can then derive the distribution function for an ensemble of species with a range of masses.

The generalized interaction potential between particles (galaxies) each of which has an isothermal halo is given by (Ahmad et al. 2002)

$$
\phi(r)=-\frac{G m^{2}}{\left(r^{2}+\epsilon^{2}\right)^{1 / 2}}
$$

where $G$ is the gravitational constant, $r$ is the separation between a pair of particles, $m$ is the mass of each particle, and $\epsilon$ is a parameter related to the finite size of a galaxy in proper coordinates. We can generalize this interaction potential by factoring out the $\epsilon$ terms to get

$$
\phi(r)=-\frac{G m^{2}}{r} \kappa(\epsilon / r)
$$


where $\kappa(\epsilon / r)$ represents a modification to the Newtonian potential. This modification only affects the potential energy part of the configuration integral given by equation (3) of Ahmad et al. (2002)

$$
Q_{N}(T, V)=\int \ldots \int \exp \left[-\phi\left(\mathbf{r}_{1}, \mathbf{r}_{2}, \ldots, \mathbf{r}_{n}\right) T^{-1}\right] d^{3 N} \mathbf{r}
$$

where $T$ is the kinetic temperature of the ensemble in units where Boltzmann's constant is unity, and $\phi$ is the interparticle potential energy of the ensemble. By following the procedure in section 2 of Ahmad et al. (2002), the potential energy part of the Hamiltonian for a 2-galaxy system becomes

$$
Q_{2}(T, V)=V^{2}\left[1+\frac{3 G m^{2}}{2 T(\bar{n})^{-1 / 3}} \zeta\left(\frac{\epsilon}{R_{1}}\right)\right]
$$

where $R_{1}$ is the scale where the two-galaxy correlation function is negligible, $\bar{n}$ is the number of particles per unit volume, and

$$
\zeta\left(\frac{\epsilon}{R_{1}}\right)=\int_{0}^{R_{1}} \frac{2 r}{R_{1}^{2}} \kappa\left(\frac{\epsilon}{r}\right) d r
$$

describes how a modification to the potential changes the partition function.

The modification given by equation (6) is analogous to $\alpha\left(\epsilon / R_{1}\right)$ given by equation (16) of Ahmad et al. (2002), but can describe a generalized modification of the potential rather than the particular modification that arises from an isothermal halo. The effects of a modified potential enter into the distribution function only through the parameter $b$, which now becomes

$$
b_{\epsilon}=\frac{(3 / 2) G^{3} m^{6} \bar{n} T^{-3} \zeta\left(\epsilon / R_{1}\right)}{1+(3 / 2) G^{3} m^{6} \bar{n} T^{-3} \zeta\left(\epsilon / R_{1}\right)} .
$$

For an attractive potential, $\kappa(\epsilon / r)$ is is always positive and hence $\zeta\left(\epsilon / R_{1}\right)>0$ for all values of $R_{1}$ so from equation (77), $0 \leq b_{\epsilon} \leq 1$.

From the thermodynamic variables of the system given by equations (26)-(30) of Ahmad et al. (2002), we see that the forms of the distribution function $f_{V}(N)$ and the thermodynamic functions of the system are essentially unchanged by a modified potential although their values are different. This shows that the form of the galaxy distribution function is robust to modified potentials. This also allows us to treat merging galaxy pairs as a separate species and extend the GQED to describe the distribution of merging galaxy pairs.

A merging galaxy pair which is bound will have its dynamical center at its center-of-mass, and will relax to form a single galaxy at its center-of-mass. Hence the position of the centers-of-mass of these merging pairs will be related to the positions of the merged galaxies. While they merge, these merging pairs form an extended system with an external potential given by the vector sum of the external potentials of both galaxies in the merging pair:

$$
\phi_{2}(\mathbf{r})=-\frac{G m_{1} M}{\left\|\mathbf{r}+\mathbf{x}_{1}\right\|}-\frac{G m_{2} M}{\left\|\mathbf{r}+\mathbf{x}_{2}\right\|}=-\frac{G m^{2}}{\|\mathbf{r}\|}\left(\frac{\|\mathbf{r}\|}{\left\|\mathbf{r}+\mathbf{x}_{1}\right\|}+\frac{\|\mathbf{r}\|}{\left\|\mathbf{r}+\mathbf{x}_{2}\right\|}\right)
$$


where $\mathbf{r}$ is the distance from the center of mass of the merging pair to a more distant galaxy of mass $M$, and $\mathbf{x}_{1}$ and $\mathbf{x}_{2}$ are the distances from each component to the center-of-mass of the system. To approximate the case when mergers between similar-sized galaxies have the dominant effect on the distribution function, we assume that all galaxies have the same mass $m$ so that $m=m_{1}=m_{2}=M$. From equation (8), the first order approximation to the external potential contains a factor of $2 m$. In the case where $\|\mathbf{r}\| \gg\|\mathbf{x}\|$, the first order term dominates and we obtain the modification factor $\kappa_{2} \approx 2$ to the potential that arises from treating these merging pairs as single extended particles.

The universe however does not contain only merging pairs. To model the presence of individual galaxies that are not currently merging, we consider a two-species distribution (Ahmad et al. 2006a) where one species consists of merging pairs, and the other species consists of galaxies that are not merging. For simplicity we consider a system containing two species of different extended particles where species 1 represents individual galaxies with an average mass of $m$, each with a halo, and species 2 represents bound pairs of merging galaxies with a total mass of $2 m$. Using the modification to the GQED described above, the potential between a pair of particles of species 1 (each of which is a galaxy) is

$$
\phi_{1}(r)=\frac{G m_{1}^{2}}{r} \kappa_{1}\left(\epsilon_{1} / r\right)
$$

where $\kappa_{1}\left(\epsilon_{1} / r\right)$ is a softening factor that arises from an extended halo with a physical extent described by $\epsilon_{1}$ (e.g. isothermal halo (Ahmad et al. 2002)). Likewise, the external potential between a particle of species 1 (a galaxy) and a particle of species 2 (a bound merging pair) is given by

$$
\phi_{2}(r)=\frac{G m_{1} m_{2}}{r} \kappa_{2}\left(\epsilon_{2} / r\right)
$$

where $m_{2}=2 m_{1}$ because there are two galaxies of mass $m_{1}$ in each merging pair, and the modification to the potential is given by $\kappa_{2}\left(\epsilon_{2} / r\right)$. Since a modified potential only changes $b$ in the single-species distribution function, a modified potential only changes the two-species clustering parameter $b_{m}$ in the two-species distribution function

$$
b_{m}=\frac{N_{1}}{N} \frac{\beta \bar{n} T^{-3}}{1+\beta \bar{n} T^{-3}}+\frac{N_{2}}{N} \frac{\beta_{12} \bar{n} T^{-3}}{1+\beta_{12} \bar{n} T^{-3}}=\frac{b}{1+N_{2} / N_{1}}\left(1+\frac{\left(N_{2} / N_{1}\right)\left(\beta_{12} / \beta\right)}{1-b+\left(\beta_{12} / \beta\right) b}\right)
$$

where $\beta$ and $\beta_{12}$ are given by

$$
\begin{aligned}
\beta & =\frac{3}{2}\left(G m_{1}^{2}\right)^{3} \zeta_{2}\left(\frac{\epsilon_{1}}{R_{1}}\right)=\frac{3}{2}\left(G m_{1}^{2}\right)^{3} \int_{0}^{R_{1}} \frac{2 r}{R_{1}^{2}} \kappa_{1}\left(\frac{\epsilon_{1}}{r}\right) d r \\
\beta_{12} & =\frac{3}{2}\left(G m_{1} m_{2}\right)^{3} \zeta_{2}\left(\frac{\epsilon_{2}}{R_{1}}\right)=\frac{3}{2}\left(G m_{1} m_{2}\right)^{3} \int_{0}^{R_{1}} \frac{2 r}{R_{1}^{2}} \kappa_{2}\left(\frac{\epsilon_{2}}{r}\right) d r
\end{aligned}
$$

and $b$ is the single-species clustering parameter given by (Ahmad et al. 2002)

$$
b=\frac{\beta \bar{n} T^{-3}}{1+\beta \bar{n} T^{-3}} .
$$


The two-species distribution function is thus (Ahmad et al. 2006a)

$$
\begin{aligned}
f_{V}(N)= & \frac{\bar{N}(1-b)}{N !}[\bar{N}(1-b)+N b]^{N_{1}-1}\left[\frac{\bar{N}(1-b)+\left(\beta_{12} / \beta\right) N b}{1-b+\left(\beta_{12} / \beta\right) b}\right]^{N_{2}} \\
& \times \exp \left[-\bar{N}\left(1-b_{m}\right)-N b_{m}\right] .
\end{aligned}
$$

The two-species distribution reduces to the single-species distribution function in the limit $N_{2} / N_{1} \rightarrow$ 0 and $\beta_{12} / \beta \rightarrow 1$. When $N_{1} \gg N_{2}$ and $\bar{N}$ is large, the deviation from the GQED is small because

the $[\bar{N}(1-b)+N b]^{N_{1}-1}$ term dominates. Measurements of the fraction of merging pairs in the VVDS catalog (de Ravel et al. 2009) suggest that for redshifts of $z \lesssim 1, N_{2} / N_{1} \approx 10 \%$. Assuming these mergers are between galaxies that are similar in mass, $m_{2} \approx 2 m_{1}$ so $\beta_{12} / \beta \approx 8$. For large cells which are a representative sample of the universe, $\bar{N} \gtrsim 100$ and the difference between the two-species distribution and the single-species distribution is small on the level of about $5 \%$. Hence under these conditions, the only significant effect of galaxy mergers in this context is a change in the average mass of a galaxy.

\section{Redshift evolution of $b$}

To determine the change in the clustering parameter $b$ we consider a merging pair of galaxies each of mass $m$ which approach each other with velocities of $\mathbf{v}_{1}$ and $\mathbf{v}_{2}$. Since momentum is conserved, the merged galaxy follows the trajectory of the center of mass of the progenitors, and has a final velocity after the merger of $\mathbf{v}_{f}=\left(\mathbf{v}_{1}+\mathbf{v}_{2}\right) / 2$. The final velocity of the merged galaxy depends on the detailed dynamics of the system, but by averaging over all orientations, we find that mergers will not change the average kinetic energy of an ensemble. Hence the more important contribution to the evolution of $b$ comes from the change in the positions of galaxies and the expansion of the universe.

We extend the analysis of Saslaw (1992) to describe an ensemble where galaxies merge by considering the effect of the adiabatic expansion of the universe. The equations of state for internal energy $U$ and pressure $P$ are (Saslaw 2000; Ahmad et al. 2002)

$$
U=\frac{3}{2} \bar{N} T(1-2 b)
$$

and

$$
P=\frac{\bar{N} T}{V}(1-b)
$$

where $V$ is the volume and $T$ is the kinetic temperature of peculiar velocities in units where the Boltzmann constant is 1. Equations (14) and (17) can be combined to get (Saslaw 1992)

$$
b=\frac{b_{0} \bar{n} T^{-3}}{1+b_{0} \bar{n} T^{-3}}=b_{0} P T^{-4} .
$$


The analysis of section 2 generalizes equation (18) to extended objects with the generalized form of $b$ given in equation (77). By comparing equations (18) and (7) we see that $b_{0}$ is given by

$$
b_{0}=\frac{3}{2} G^{3} \bar{m}^{6} \zeta\left(\frac{\epsilon}{R_{1}}\right)
$$

where $\bar{m}$ is the average mass of a galaxy, and $\zeta$ is a function of order unity that depends weakly on $\epsilon / R_{1}$.

Because $R_{1}$ is defined as the scale where the two-galaxy correlation function becomes negligible, the universe is approximately uniform averaged over scales $\gtrsim R_{1}$. Here we take $R_{1}$ to be the scale at which the two-galaxy correlation function begins to decrease faster than a power law. Measurements from the 2DFGRS (Hawkins et al. 2003) have indicated that $R_{1}$ is about $12 h^{-1} \mathrm{Mpc}$ at which the two-galaxy correlation function is of the order $10^{-2}$. Here $h=H_{0} / 100$ is the reduced Hubble constant. We note that such cells are large enough to contain individual field galaxies and clusters of galaxies, and hence would be an approximately representative sample of the universe. Assuming that on such scales, galaxies have isotropic average velocities, then for cells with a radius larger than $R_{1}$, galaxies are as likely to enter a cell as they are to leave a cell. With this assumption, the total mass in each comoving cell $M_{c}$ would be approximately constant, and each cell would have on average $\bar{N}=M_{c} / \bar{m}$ galaxies and $d(\bar{m} \bar{N})=0$. Therefore $\bar{N} \propto \bar{m}^{-1}$ and $b_{0}$ can be written as a function of $\bar{N}$ instead of $\bar{m}$. This transforms equation (18) into the form

$$
b=b_{0}(\bar{N}) P T^{-4}=\frac{3}{2} G^{3}\left(\frac{M_{c}}{\bar{N}}\right)^{6} \zeta\left(\frac{\epsilon}{R_{1}}\right) P T^{-4} .
$$

Differentiating equation (20) with respect to $\bar{N}$ gives

$$
\frac{d b}{b}=-6 \frac{d \bar{N}}{\bar{N}}\left(1+\frac{\bar{N}}{6 R_{1}}\left(\frac{\partial \ln \zeta\left(\epsilon / R_{1}\right)}{\partial \bar{N}}\right)_{T, P}\right)=-6 \frac{d \bar{N}}{\bar{N}}\left(1+\zeta_{\star}\right)
$$

from which we define the term

$$
\zeta_{\star}=\frac{\bar{N}}{6 R_{1}}\left(\frac{\partial \ln \zeta\left(\epsilon / R_{1}\right)}{\partial \bar{N}}\right)_{T, P}
$$

In general, we do not rule out the possibility that $\zeta\left(\epsilon / R_{1}\right)$ may indirectly depend on $\bar{N}$, hence the $\partial \ln \zeta\left(\epsilon / R_{1}\right) / \partial \bar{N}$ factor in $\zeta_{\star}$ may be nonzero.

In the case of adiabatic expansion, equations (16) and (17), give

$$
0=d U+P d V=\frac{3}{2}(1-2 b)\left[T d \bar{N}+\left.\bar{N} d T\right|_{\bar{N}, P}\right]-3 \bar{N} T d b+\bar{N} T(1-b) \frac{d V}{V} .
$$

Equation (20) implies

$$
\left.d T\right|_{\bar{N}, P}=-\frac{T d b}{4 b}
$$


and hence using $d V / V=3 d R / R$ where $R$ is the scale length of the universe, we have

$$
0=\frac{3}{2}(1-2 b) T d \bar{N}-\frac{3}{2}(1-2 b) \bar{N} T \frac{d b}{4 b}-3 \bar{N} T d b+3 \bar{N} T(1-b) \frac{d R}{R} .
$$

Rearranging the terms and using equation (21), we find in terms of redshift $z \propto 1 / R-1$

$$
\frac{d b}{d z}=-\frac{1-b}{1+z}\left(\frac{1+6 b}{8 b}+\frac{1-2 b}{12 b \zeta_{\star}}\right)^{-1} .
$$

To illustrate how mergers contribute to the time evolution of $b$, we consider a simple model of a galaxy. In our model, galaxies have isothermal halos with a characteristic radius $\epsilon$, and all galaxies have the same density so that in a cell of total mass $M_{c}$

$$
\epsilon=a(\bar{m})^{1 / 3}=a\left(\frac{M_{c}}{\bar{N}}\right)^{1 / 3}
$$

for some constant of proportionality $a$ such that $\epsilon$ depends on the average mass of a galaxy. We use the GQED and form of $\zeta$ for such a case from Ahmad et al. (2002) to obtain the constraint $-1 / 18 \leq \zeta_{\star} \leq 0$. The extremes of this constraint gives

$$
\frac{d b}{d z}=-\frac{1-b}{1+z}\left(\frac{24 b}{5+14 b}\right)
$$

for the case where $\zeta_{\star}=0$ and

$$
\frac{d b}{d z}=-\frac{1-b}{1+z}\left(\frac{136 b}{29+78 b}\right)
$$

for the case with $\zeta_{\star}=-1 / 18$ with all other cases occurring in between equations (28) and (29).

Since $0 \leq b \leq 1$, we compare the two cases numerically and find that the difference between the two cases is small at less than $2 \%$. This result tells us that although galaxy mergers can influence the time evolution of $b$, their influence mostly results from changes of the number of galaxies in a cell.

\section{Conclusion and Future Work}

We have established that the effects of galaxy mergers leave the form of the galaxy distribution function essentially unchanged and just alter the parameters of the counts in cells distribution. In particular, by describing bound merging pairs as objects with a modified gravitational potential, we obtain a modified form of the two-species counts in cells distribution (Ahmad et al. 2006a) and show that it only changes the counts in cells distribution slightly from the single-species result given by equation (11).

As a result of mergers, the clustering parameter $b$ increases with time, and we have shown that it depends very weakly on the physical extent of a galaxy and the scale $R_{1}$ at which the two point 
correlation function is negligible. The effect of the physical extent of a galaxy changes $d b / d z$ by less than $2 \%$, which shows that the evolution of $b$ depends mainly on the adiabatic expansion of the universe and the change in the number of galaxies from mergers.

These results show that even when we take galaxy mergers into account, we can not only reproduce the GQED but also trace the evolution of the clustering parameters. However, an analysis

of the GOODS catalog (Rahmani et al. 2009) indicates a large variation between the North and South fields and suggests that the sample is probably too small to draw any meaningful conclusions about the evolution of $b$ at high redshift. Future surveys however may provide sufficiently large samples at high redshifts to test our predicted evolution of $b$.

\section{Acknowledgments}

A. Yang is grateful for the support from the National University of Singapore and the Institute of Astronomy of the University of Cambridge where part of this work was done.

A. H. Chan would like to thank the department of History \& Philosophy of Science, Cambridge University and Nanyang Polytechnic for kind hospitality where part of the initial work was done.

\section{REFERENCES}

Ahmad, F., Malik, M. A., and Masood, S. 2006a, Intl. J. Modern Physics D, 15, 1267

Ahmad, F., Saslaw, W. C., and Bhat, N. I. 2002, Astrophys. J., 571, 576

Ahmad, F., Saslaw, W. C., and Malik, M. A. 2006b, Astrophys. J., 645, 940

de Ravel, L. et al. 2009, Astronomy \& Astrophysics, 498, 379

Fry, J. N. 1986, Astrophys. J., 306, 358

Hawkins, E. et al. 2003, Monthly Notices of the Royal Astronomical Society, 346, 78

Leong, B. and Saslaw, W. C. 2004, Astrophys. J., 608, 636

Rahmani, H., Saslaw, W. C., and Tavasoli, S. 2009, Astrophys. J., 695, 1121

Saslaw, W. C. 1992, Astrophys. J., 391, 423

—. 2000, The distribution of the galaxies : gravitational clustering in cosmology (Cambridge, UK: Cambridge University Press)

Saslaw, W. C., Chitre, S. M., Itoh, M., and Inagaki, S. 1990, Astrophys. J., 365, 419 
Saslaw, W. C. and Yang, A. 2010, in Lecture Notes of the Les Houches Summer School: LongRange Interacting Systems, ed. T. Dauxois, S. Ruffo, \& L. F. Cugliandolo, Vol. XC (Oxford, UK: Oxford University Press), 377-398

Sivakoff, G. R. and Saslaw, W. C. 2005, Astrophys. J., 626, 795

This preprint was prepared with the AAS $\mathrm{LAT}_{\mathrm{E}} \mathrm{X}$ macros v5.2. 